\title{
On the rank of $p$-schemes
}

\author{
Fatemeh Raei Barandagh \\ Department of Mathematics \\ K. N. Toosi University of Technology \\ Tehran, Iran \\ f.raei@dena.kntu.ac.ir
}

\author{
Amir Rahnamai Barghi* \\ Department of Mathematics \\ K. N. Toosi University of Technology \\ Tehran, Iran \\ and School of Mathematics and Statistics \\ Carleton University \\ Ottawa, Canada \\ rahnama@kntu.ac.ir
}

Submitted: Jan 27, 2013; Accepted: May 6, 2013; Published: May 16, 2013

Mathematics Subject Classifications: 05E30, 20E22

\begin{abstract}
Let $n>1$ be an integer and $p$ be a prime number. Denote by $\mathfrak{C}_{p^{n}}$ the class of non-thin association $p$-schemes of degree $p^{n}$. A sharp upper and lower bounds on the rank of schemes in $\mathfrak{C}_{p^{n}}$ with a certain order of thin radical are obtained. Moreover, all schemes in this class whose rank are equal to the lower bound are characterized and some schemes in this class whose rank are equal to the upper bound are constructed. Finally, it is shown that the scheme with minimum rank in $\mathfrak{C}_{p^{n}}$ is unique up to isomorphism, and it is a fusion of any association $p$-schemes with degree $p^{n}$.
\end{abstract}

Keywords: association scheme, thin radical, wreath product

\section{Introduction}

Towards generalization of the theory of finite groups to the theory of schemes or coherent configurations, it is quite natural question which schemes correspond to $p$-groups, where $p$ is a prime number. In this direction, the concept of $p$-schemes was given in [3] as follows: a scheme $\mathcal{C}$ is called $p$-scheme if the cardinality of each basis relation of $\mathcal{C}$ is a power of $p$. Recently some algebraic and combinatorial properties of this notion were studied in [4], [5] and [1].

In this paper we focus on association $p$-schemes and refer them as $p$-schemes. Let $n$ be an integer. The class of thin $p$-schemes of degree $p^{n}$ are denoted by $\mathcal{T}_{p^{n}}$. All $p$-schemes

${ }^{*}$ Part of this work has been done while the author was visiting the School of Mathematics and Statistics, Carleton University as an Adjunct Research Professor. 
on $p$ points are thin, thus they are unique up to isomorphism, we denote it by $T_{p}$. For $n>1$, the class of non-thin $p$-schemes of degree $p^{n}$ are denoted by $\mathfrak{C}_{p^{n}}$. Given a scheme in $\mathcal{T}_{p^{n}}$, the rank of scheme is equal to degree of scheme. We will determine upper and lower bounds on the rank of $p$-schemes in $\mathfrak{C}_{p^{n}}$.

Suppose that $\mathcal{C} \in \mathfrak{C}_{p^{n}}$, this implies that the thin radical $\mathcal{O}_{\vartheta}(\mathcal{C})$ of $\mathcal{C}$ is a non-trivial $p$-group [7]. Thus the order of the thin radical is a power of $p$. The following theorems are the main results of this paper.

Theorem 1. Given integers $n$ and $t, 1 \leqslant t<n$, let $\mathbf{l}(t, p, n)$ and $\mathbf{u}(t, p, n)$ be the minimum and maximum rank of schemes with thin radical of order $p^{t}$ in $\mathfrak{C}_{p^{n}}$, respectively. Then

$$
\mathbf{l}(t, p, n)=p^{t}+(n-t)(p-1), \quad \mathbf{u}(t, p, n)=p^{n-1}+p^{t-1}(p-1) .
$$

Remark 2. The minimum and the maximum rank for $p$-schemes in $\mathfrak{C}_{p^{n}}$ is given where $t=1$ and $t=n-1$, respectively. Thus

$$
\begin{aligned}
\mathbf{l}(p, n) & :=\min \left\{\operatorname{rk}(\mathcal{C}): \mathcal{C} \in \mathfrak{C}_{p^{n}}\right\}=n(p-1)+1, \\
\mathbf{u}(p, n) & :=\max \left\{\operatorname{rk}(\mathcal{C}): \mathcal{C} \in \mathfrak{C}_{p^{n}}\right\}=2 p^{n-1}-p^{n-2} .
\end{aligned}
$$

Especially, in Theorem 7 we characterize $p$-schemes with $\operatorname{rank} \mathbf{l}(t, p, n)$. It follows that the $p$-schemes with minimum rank in $\mathfrak{C}_{p^{n}}$ are isomorphic to the wreath product of $n$ copies of $T_{p}$, which denoted by $\left(T_{p}\right)^{2 n}$. Moreover, we prove the following theorem:

Theorem 3. Any p-scheme with degree $p^{n}$ is isomorphic to a fission of $\left(T_{p}\right)^{2 n}$.

This paper is organized as follows. In Section 2, we present some notations and terminology on association schemes. In Section 3, we review some basic results on $p$ schemes. In Section 4, we prove our main theorems.

\section{Background on association schemes}

\section{1}

Let $V$ be a non-empty finite set and let $\mathcal{R}=\left\{R_{0}, R_{1}, \ldots, R_{d}\right\}$ be a set of non-empty binary relations on $V$ that partitions $V \times V$, where $R_{0}=\Delta(V)$, the set of all pairs $(v, v)$ with $v \in V$. The pair $\mathcal{C}=(V, \mathcal{R})$ is called an association scheme (or simply scheme) if for every $R_{i} \in \mathcal{R}, R_{i}^{\mathrm{t}}:=\left\{(u, v):(v, u) \in R_{i}\right\} \in \mathcal{R}$ so denote $R_{i}^{\mathrm{t}}$ by $R_{i^{\prime}}$, and for all $R_{i}, R_{j}, R_{k} \in \mathcal{R}$ there exists an intersection number $p_{i j}^{k}$ such that $p_{i j}^{k}=\left|R_{i}(u) \cap R_{j^{\prime}}(v)\right|$ for all $(u, v) \in R_{k}$, where $R(u)$ is the set of all elements $v \in V$ with $(u, v) \in R$ for each $R \in \mathcal{R}$.

Observe that $p_{i j}^{0}=\left|R_{i}(u) \cap R_{j^{\prime}}(u)\right|$ for all $u \in V$. In particular, $p_{i j}^{0}$ is zero unless $R_{i}=R_{j^{\prime}}$. The elements of $V$ are called points and those of $\mathcal{R}$ are called basis relations of $\mathcal{C}$. The numbers $|V|$ and $|\mathcal{R}|$ are called the degree and the rank of $\mathcal{C}$, and are denoted by $\operatorname{deg}(\mathcal{C})$ and $\operatorname{rk}(\mathcal{C})$, respectively. We write $\mathrm{d}\left(R_{i}\right)=p_{i i^{\prime}}^{0}$, and refer to this number as the 
valency of $R_{i}$. The $d+1$ relations $R_{i}$ are conveniently described by their $\{0,1\}$-adjacency matrices $A_{0}, A_{1}, \ldots, A_{d}$ defined by $\left(A_{i}\right)_{u v}=1$ if $(u, v) \in R_{i} ; 0$ otherwise. Denote by $\mathcal{R}^{\cup}$ the set of all unions of the elements of $\mathcal{R}$.

Let $\mathcal{S}$ and $\mathcal{Q}$ be non-empty subsets of $\mathcal{R}$. We define $\mathcal{S} \mathcal{Q}$ to be the set of all basis relations $R_{k}$ in $\mathcal{R}$ for which there exists $R_{i}$ in $\mathcal{S}$ and $R_{j}$ in $\mathcal{Q}$ satisfying $p_{i j}^{k} \neq 0$. If $\mathcal{S}=\left\{R_{i}\right\}$ and $\mathcal{Q}=\left\{R_{j}\right\}$, we write $R_{i} R_{j}$ instead of $\mathcal{S} \mathcal{Q}$. The set $\mathcal{S} \mathcal{Q}$ is called the complex product of $\mathcal{S}$ and $\mathcal{Q}$.

A scheme $\mathcal{C}=(V, \mathcal{R})$ is said to be schurian if there exists a transitive permutation group $G \leqslant \operatorname{Sym}(V)$ such that the set of 2 -orbits of $G$ is equal to $\mathcal{R}$.

\section{2}

Let $\mathcal{C}=(V, \mathcal{R})$ be a scheme. An equivalence relation $E$ on $V$ is called an equivalence of $\mathcal{C}$ if $E$ is a union of some basis relations of $\mathcal{C}$. Denote by $\mathcal{E}(\mathcal{C})$ the set of all equivalences of $\mathcal{C}$. For each $E \in \mathcal{E}(\mathcal{C})$ we define degree of $E, d(E)$, the sum of valency of all basis relations of $\mathcal{C}$ which lie in $E$. Let $X$ be a non-empty subset of $V$ and let $E$ be an equivalence of the scheme $\mathcal{C}$. Denote by $X / E$ the set of classes of the equivalence relation $E \cap(X \times X)$, and $R_{X / E}$ the set of pairs $(Y, Z)$ in $(X / E) \times(X / E)$ such that $R_{Y, Z} \neq \emptyset$, where $R_{Y, Z}=R \cap(Y \times Z)$. Also denote by $\mathcal{R}_{X / E}$ the set of all non-empty relations $R_{X / E}$ on $X / E$ where $R \in \mathcal{R}$. Then the pair

$$
\mathcal{C}_{X / E}=\left(X / E, \mathcal{R}_{X / E}\right)
$$

is a scheme. If $X \in V / E$, then the pair $\mathcal{C}_{X}=\left(X, \mathcal{R}_{X}\right)$ is a scheme.

\section{3}

Let $\mathcal{C}=(V, \mathcal{R})$ be a scheme. The set

$$
\mathcal{O}_{\vartheta}(\mathcal{C})=\{R \in \mathcal{R}: d(R)=1\}
$$

is called the thin radical of $\mathcal{C}$. It is clear that $\bigcup_{R \in \mathcal{O}_{\vartheta}(\mathcal{C})} R \in \mathcal{E}(\mathcal{C})$.

\section{4}

Two schemes $\mathcal{C}$ and $\mathcal{C}^{\prime}$ are called isomorphic if there exists a bijection between their point sets which induces a bijection between their sets of basis relations, and we denote it by $\mathcal{C} \simeq \mathcal{C}^{\prime}$. Such a bijection is called an isomorphism between $\mathcal{C}$ and $\mathcal{C}^{\prime}$.

\section{5}

There is a partial order on the set of all schemes on set $V$. Given two schemes $\mathcal{C}=(V, \mathcal{R})$ and $\mathcal{C}^{\prime}=\left(V, \mathcal{R}^{\prime}\right)$ we define $\mathcal{C} \leqslant \mathcal{C}^{\prime}$ if and only if $\mathcal{R}^{\cup} \subseteq\left(\mathcal{R}^{\prime}\right)^{\cup}$. In this case $\mathcal{C}$ is called a fusion of $\mathcal{C}^{\prime}$ and also $\mathcal{C}^{\prime}$ is called a fission of $\mathcal{C}$. The minimal element with respect to that order is trivial scheme, the scheme with the set of basis relations $\left\{\Delta(V), V^{2} \backslash \Delta(V)\right\}$. 


\section{6}

Given non-empty finite sets $V_{1}, V_{2}$, denote by $R_{1} \otimes R_{2}$ the set of elements $\left((u, v),\left(u^{\prime}, v^{\prime}\right)\right)$ in $\left(V_{1} \times V_{2}\right) \times\left(V_{1} \times V_{2}\right)$ such that $\left(u, u^{\prime}\right) \in R_{1}$ and $\left(v, v^{\prime}\right) \in R_{2}$. Let $\mathcal{C}_{i}=\left(V_{i}, \mathcal{R}_{i}\right), i=1,2$, be two schemes. Denote by $\mathcal{C}_{1} \otimes \mathcal{C}_{2}$ the set of all relations $R_{1} \otimes R_{2}$ where $R_{1} \in \mathcal{R}_{1}$ and $R_{2} \in \mathcal{R}_{2}$. Then the pair

$$
\mathcal{C}_{1} \otimes \mathcal{C}_{2}=\left(V_{1} \times V_{2}, \mathcal{R}_{1} \otimes \mathcal{R}_{2}\right)
$$

is a scheme and is called the tensor product of $\mathcal{C}_{1}$ and $\mathcal{C}_{2}$. It is easily seen that

$$
\operatorname{deg}\left(\mathcal{C}_{1} \otimes \mathcal{C}_{2}\right)=\operatorname{deg}\left(\mathcal{C}_{1}\right) \operatorname{deg}\left(\mathcal{C}_{2}\right), \quad \operatorname{rk}\left(\mathcal{C}_{1} \otimes \mathcal{C}_{2}\right)=\operatorname{rk}\left(\mathcal{C}_{1}\right) \operatorname{rk}\left(\mathcal{C}_{2}\right)
$$

\section{7}

Let $\mathcal{C}_{i}=\left(V_{i}, \mathcal{R}_{i}\right), i=1,2$ be two schemes, where $V_{1}$ and $V_{2}$ are disjoint. Let

$$
\mathcal{R}_{1}\left\langle\mathcal{R}_{2}=\left\{\Delta\left(V_{2}\right) \otimes R: R \in \mathcal{R}_{1}\right\} \cup\left\{S \otimes V_{1} \times V_{1}: S \in \mathcal{R}_{2} \backslash \Delta\left(V_{2}\right)\right\} .\right.
$$

Then the pair $\mathcal{C}_{1} \prec \mathcal{C}_{2}=\left(V_{1} \times V_{2}, \mathcal{R}_{1} \prec \mathcal{R}_{2}\right)$ is a scheme and is called the wreath product of $\mathcal{C}_{1}$ by $\mathcal{C}_{2}$. Moreover, let $\mathcal{R}_{1}=\left\{R_{0}, R_{1}, \ldots, R_{d}\right\}, \mathcal{R}_{2}=\left\{S_{0}, S_{1}, \ldots, S_{e}\right\}$; and also let $A_{0}, A_{1}, \ldots, A_{d}$ and $B_{0}, B_{1}, \ldots, B_{e}$ be the adjacency matrices of $R_{i}$ and $S_{j}$, respectively, $0 \leqslant i \leqslant d$ and $0 \leqslant j \leqslant e$. Then the elements of $V_{1} \times V_{2}$ can be ordered such that the adjacency matrices of $\mathcal{C}_{1} \prec \mathcal{C}_{2}$ are given by

$$
\begin{array}{r}
C_{0}=B_{0} \otimes A_{0}, C_{1}=B_{0} \otimes A_{1}, \ldots, C_{d}=B_{0} \otimes A_{d}, \\
C_{d+1}=B_{1} \otimes J_{\left|V_{1}\right|}, \ldots, C_{d+e}=B_{e} \otimes J_{\left|V_{1}\right|},
\end{array}
$$

where $J_{n}$ is the $n \times n$ matrix whose entries are all 1 . From definition of the wreath product it follows that

$$
\operatorname{deg}\left(\mathcal{C}_{1} \prec \mathcal{C}_{2}\right)=\operatorname{deg}\left(\mathcal{C}_{1}\right) \operatorname{deg}\left(\mathcal{C}_{2}\right), \quad \operatorname{rk}\left(\mathcal{C}_{1} \prec \mathcal{C}_{2}\right)=\operatorname{rk}\left(\mathcal{C}_{1}\right)+\operatorname{rk}\left(\mathcal{C}_{2}\right)-1
$$

Note that $\mathcal{C}_{1} \prec \mathcal{C}_{2}$ is schurian if and only if $\mathcal{C}_{1}$ and $\mathcal{C}_{2}$ are schurian. It is well known that $E=\bigcup_{v \in V_{2}} V_{1} \times\{v\}$ is an equivalence of $\mathcal{C}=\mathcal{C}_{1} \prec \mathcal{C}_{2}$ such that $\mathcal{C}_{X} \simeq \mathcal{C}_{1}$ for all $X \in V / E$ and $\mathcal{C}_{V / E} \simeq \mathcal{C}_{2}$.

For a given scheme $\mathcal{C}$ and a natural number $m$, we define the wreath power $(\mathcal{C})^{2 m}:=$ $\mathcal{C} \imath \mathcal{C} \imath \ldots$ C , the wreath product of $m$ copies of $\mathcal{C}$.

\section{$3 \quad p$-schemes}

Fix a prime number $p$. A scheme $\mathcal{C}$ is called $p$-scheme if the cardinality of each basis relation of $\mathcal{C}$ is a power of $p$. Thus the degree of any $p$-scheme is a power of $p$, and $\mathcal{O}_{\vartheta}(\mathcal{C})$ is a non-trivial $p$-group with respect to complex product. Thus there is a positive integer $m(\mathcal{C})$ such that the order of thin radical is $p^{m(\mathcal{C})}$. So there exists non-diagonal relation 
$R \in \mathcal{O}_{\vartheta}(\mathcal{C})$ such that $d(R)=1$. The smallest equivalence $\langle R\rangle$ of $\mathcal{C}$ containing $R$ is the union of relations $R^{i}, i=0,1, \ldots, p-1$. This implies that the scheme $\mathcal{C}_{X}$ is a thin scheme on $p$ points, for any $X \in V /\langle R\rangle$. In [7, Corollary 5.6.8] it is proved that $\mathcal{C}=(V, \mathcal{R})$ is a $p$-scheme if and only if there exists a sequence of equivalences of the scheme $\mathcal{C}$,

$$
\Delta(V)=E_{0} \subset E_{1} \subset \ldots \subset E_{m}=V \times V
$$

such that $E_{i} \in \mathcal{E}(\mathcal{C})$ and $\mathcal{C}_{X / E_{i}}$ is a thin scheme on $p$ points for $i=0,1, \ldots, m-1$ and all $X \in V / E_{i+1}$, we denote such a sequence by $\left\{E_{i}\right\}_{i=0}^{m}$. The set of all these sequences denoted by $\mathrm{PE}(\mathcal{C})$.

For each $\left\{E_{i}\right\}_{i=0}^{m} \in \operatorname{PE}(\mathcal{C})$ clearly $d\left(E_{1}\right)=p$. Since $\mathcal{C}_{X / E_{i}}$ has $p$ points, for any $X \in V / E_{i+1}$ it follows that $d\left(E_{i+1}\right)=p d\left(E_{i}\right)$, where $0 \leqslant i \leqslant m-1$. This implies that $m=n$ and $d\left(E_{i}\right)=p^{i}$, for $0 \leqslant i \leqslant n$. Therefore, we proved the following lemma.

Lemma 4. Let $\mathcal{C}$ be a $p$-scheme on $p^{n}$ points and let $\left\{E_{i}\right\}_{i=0}^{m} \in \operatorname{PE}(\mathcal{C})$. Then $m=n$ and

$$
d\left(E_{i}\right)=p^{i}, \text { for each } i, \quad 0 \leqslant i \leqslant n .
$$

Let $\mathcal{C}=(V, \mathcal{R})$ be a scheme with $\mathcal{R}=\left\{R_{0}, R_{1}, \ldots, R_{d}\right\}$. The valency pattern of $\mathcal{C}$ is the multiset $\left\{n_{i} \mid 0 \leqslant i \leqslant d\right\}$ denoted by

$$
\operatorname{VP}(\mathcal{C})=\left(\begin{array}{cccc}
n_{0} & n_{1} & \ldots & n_{s} \\
t_{0} & t_{1} & \ldots & t_{s}
\end{array}\right)
$$

where $n_{0}<n_{1}<\ldots<n_{s}$ are the distinct valencies and $t_{j}$ 's are multiplicities of $n_{j}$, i.e.,

$$
t_{j}=\left|\left\{i \in\{0,1, \ldots, d\}: d\left(R_{i}\right)=n_{j}\right\}\right| .
$$

Let $\mathcal{C}$ be a $p$-scheme on $p^{n}$ points with $\left\{E_{i}\right\}_{i=0}^{n} \in \operatorname{PE}(\mathcal{C})$. As we have seen in the above, the thin radical of $\mathcal{C}$ is a $p$-group of order $p^{m(\mathcal{C})}$, where $m(\mathcal{C}) \geqslant 1$. Hence the valency pattern of $\mathcal{C}$ is as follows

$$
\operatorname{VP}(\mathcal{C})=\left(\begin{array}{ccccc}
1 & p^{s_{1}} & p^{s_{2}} & \ldots & p^{s_{k}} \\
p^{m(\mathcal{C})} & t_{1} & t_{2} & \ldots & t_{k}
\end{array}\right)
$$

where $0<s_{1}<\ldots<s_{k}<n$. Now, suppose that for all $i, 1 \leqslant i \leqslant n-m(\mathcal{C})$

$$
k=n-m(\mathcal{C}), \quad s_{i}=m(\mathcal{C})+i-1, \quad t_{i}=p-1 .
$$

From Lemma 4 , it follows that $d\left(E_{m(\mathcal{C})+j}\right)=p^{m(\mathcal{C})+j}$ for $j, 0 \leqslant j \leqslant n-m(\mathcal{C})-1$. Since $\Delta(V) \subset E_{m(\mathcal{C})}$, we conclude that

$$
d\left(E_{m(\mathcal{C})}\right)=1+\sum_{R \in \mathcal{O}_{\vartheta}} d(R)
$$


Moreover, from the inclusion $E_{m(\mathcal{C})+j} \subset E_{m(\mathcal{C})+j+1}$ it follows that

$$
d\left(E_{m(\mathcal{C})+j+1}\right)=d\left(E_{m(\mathcal{C})+j}\right)+\sum_{R \subseteq E_{m(\mathcal{C})+j+1} \backslash E_{m(\mathcal{C})+j}} d(R)
$$

On the other hand, for $R \subseteq E_{m(\mathcal{C})+j+1} \backslash E_{m(\mathcal{C})+j}$, we have $p^{m(\mathcal{C})+j-1}<d(R)<p^{m(\mathcal{C})+j+1}$. This implies that $d(R)=p^{m(\mathcal{C})+j}$. From Lemma 4 and (2) it follows that

$$
\left|\left\{R \in \mathcal{R}: R \subseteq E_{m(\mathcal{C})+j+1} \backslash E_{m(\mathcal{C})+j}, \quad d(R)=p^{m(\mathcal{C})+j}\right\}\right|=p-1 .
$$

Thus $E_{m(\mathcal{C})+j+1}=\bigcup_{d(R) \leqslant p^{m(\mathcal{C})+j}} R$. Therefore, we proved the following lemma.

Lemma 5. Let $\mathcal{C}$ be a p-scheme with $\left\{E_{i}\right\}_{i=0}^{n} \in \mathrm{PE}(\mathcal{C})$. If the valency pattern of $\mathcal{C}$ is as the following form

$$
\operatorname{VP}(\mathcal{C})=\left(\begin{array}{ccccc}
1 & p^{m(\mathcal{C})} & p^{m(\mathcal{C})+1} & \ldots & p^{n-1} \\
p^{m(\mathcal{C})} & p-1 & p-1 & \ldots & p-1
\end{array}\right)
$$

then for each $i, m(\mathcal{C}) \leqslant i \leqslant n$ we have

$$
E_{i}=\bigcup_{d(R) \leqslant p^{i-1}} R
$$

\section{Proofs of the main theorems}

\subsection{Wedge product}

In [2], Muzychuk has defined wedge product of association schemes. We construct a $p$-scheme with maximum $\operatorname{rank} \mathbf{u}(t, p, n)$ by wedge product of association schemes.

Let $\mathcal{C}=(V, \mathcal{R})$ be a association scheme such that

1) there exist $E, F \in \mathcal{E}(\mathcal{C})$, where $E \subseteq F$,

2) for each $R \in \mathcal{R}$ which $R \cap F=\emptyset$, we have $E \subseteq \operatorname{Rad}(R)$.

Where $\operatorname{Rad}(R)=\{S \in \mathcal{R}: S R=R\}$. Then we say that $\mathcal{C}$ is a wedge product of $\mathcal{C}_{X}$ and $\mathcal{C}_{V / E}$, for each $X \in V / F$. This is equivalent to Muzychuk definition of wedge product.

Example 6. Let $n>1$ be an integer and let $p$ be a prime number. Fix $t$ such that $1 \leqslant t<n$. Let $\mathcal{C}$ be the wedge product of a scheme in class $\mathcal{T}_{p^{t}}$ and a scheme in class $\mathcal{T}_{p^{n-1}}$, with two equivalences $E$ and $F$, where $d(E)=p$ and $d(F)=p^{t}$. Then $\mathcal{C} \in \mathfrak{C}_{p^{n}}$, $m(\mathcal{C})=t$ and $\operatorname{rk}(\mathcal{C})=p^{n-1}+p^{t-1}(p-1)$. 


\section{Proof of Theorem 1.}

Let $n>1$ be an integer and let $p$ be a prime number. Fix $t$ such that $1 \leqslant t<n$. Suppose that $\mathcal{C} \in \mathfrak{C}_{p^{n}}$ with the set of basis relations $\mathcal{R}$ such that $m(\mathcal{C})=t$. For $0 \leqslant j<n$, let

$$
\mathcal{R}_{j}=\left\{R \in \mathcal{R}: d(R)=p^{j}\right\}, \quad r_{j}=\left|\mathcal{R}_{j}\right|
$$

It is easily seen that

$$
\mathcal{R}=\bigcup_{j=0}^{n-1} \mathcal{R}_{j}
$$

Since the sum of all valencies of $\mathcal{C}$ is equal to $p^{n}$, from the above partition we conclude that

$$
p^{n}=\sum_{R \in \mathcal{R}} d(R)=\sum_{j=0}^{n-1} \sum_{R \in \mathcal{R}_{j}} d(R),
$$

which implies the following equality

$$
p^{n}=\sum_{k=0}^{n-1} r_{k} p^{k}=p^{t}+\sum_{k=1}^{n-1} r_{k} p^{k} .
$$

If $t=1$ then from (3), we have $p \mid 1+r_{1}$, so there is an integer $r_{1}^{\prime}$ such that $1+r_{1}=r_{1}^{\prime} p$. Now suppose that $t>1$. Then from (3), we conclude that $p \mid r_{1}$, so there is an integer $r_{1}^{\prime}$ such that $r_{1}=r_{1}^{\prime} p$, and so we have

$$
p^{t-2}+r_{1}^{\prime}+r_{2}+\sum_{k=3}^{n-1} r_{k} p^{k-2}=p^{n-2}
$$

The above equality shows that if $t=2$, then $p \mid 1+r_{1}^{\prime}+r_{2}$, so there is an integer $r_{2}^{\prime}$ such that $1+r_{1}^{\prime}+r_{2}=r_{2}^{\prime} p$. For $t>2$ we have $p \mid r_{1}^{\prime}+r_{2}$ so there is an integer $r_{2}^{\prime}$ such that $r_{1}^{\prime}+r_{2}=r_{2}^{\prime} p$. In the same way, inductively, there is an integer $r_{t}^{\prime}$ such that $1+r_{t-1}^{\prime}+r_{t}=r_{t}^{\prime} p$.

Thus, with the above explanation, for $0 \leqslant j \leqslant n-2$ we define $r_{j}^{\prime}$ as follows:

$$
r_{j}^{\prime}= \begin{cases}0, & j=0 \\ \frac{r_{j-1}^{\prime}+r_{j}}{p}, & 1 \leqslant j \leqslant n-2, \quad j \neq t \\ \frac{1+r_{t-1}^{\prime}+r_{t}}{p}, & j=t .\end{cases}
$$

Thus follow from (4), we have

$$
r_{j}= \begin{cases}r_{j}^{\prime} p-r_{j-1}^{\prime}, & 1 \leqslant j \leqslant n-2, \quad j \neq t \\ r_{t}^{\prime} p-r_{t-1}^{\prime}-1, & j=t \\ p-r_{n-2}^{\prime}, & j=n-1 .\end{cases}
$$


On the other hand, the rank of $\mathcal{C}$ is the number of basis relations of $\mathcal{C}$, so

$$
\operatorname{rk}(\mathcal{C})=\sum_{k=0}^{n-1} r_{k}=p^{t}+\sum_{k=1}^{n-1} r_{k} .
$$

Thus from (5) and (6) it follows that

$$
\begin{aligned}
\operatorname{rk}(\mathcal{C}) & =p^{t}+\sum_{j=1}^{n-2}\left(r_{j}^{\prime} p-r_{j-1}^{\prime}\right)+p-r_{n-2}^{\prime}-1 \\
& =p^{t}+(p-1)\left(\sum_{j=1}^{n-2} r_{j}^{\prime}+1\right)
\end{aligned}
$$

Now, from (3) and (4) it follows that

$$
p^{n-j}= \begin{cases}p^{t-j}+r_{j-1}^{\prime}+r_{j}+\sum_{k=j+1}^{n-1} r_{k} p^{k-j}, & 0 \leqslant j \leqslant t \\ r_{j-1}^{\prime}+r_{j}+\sum_{k=j+1}^{n-1} r_{k} p^{k-j}, & t+1 \leqslant j \leqslant n-2 .\end{cases}
$$

From (4) and (8) one can see that $r_{j}^{\prime}$ is non negative integer for all $j$ with $0 \leqslant j \leqslant t-1$ and $r_{j}^{\prime}$ is positive integer for all $j$ with $t \leqslant j \leqslant n-2$. Thus from (7) we conclude that

$$
\operatorname{rk}(\mathcal{C}) \geqslant p^{t}+(n-t)(p-1)
$$

On the other hand, let $\mathcal{C}_{1} \in \mathcal{T}_{p^{t}}$. Then the scheme $\mathcal{C}^{\prime}=\mathcal{C}_{1} 2\left(T_{p}\right)^{2(n-t)}$ belongs to $\mathfrak{C}_{p^{n}}$ with $m\left(\mathcal{C}^{\prime}\right)=t$ and its rank is equal to $p^{t}+(n-t)(p-1)$. Thus

$$
\mathbf{l}(t, p, n)=p^{t}+(n-t)(p-1) .
$$

Now, we obtain the upper bound of $\operatorname{rank} \mathcal{C} \in \mathfrak{C}_{p^{n}}$ with $m(\mathcal{C})=t$. From equation (3), we have

$$
r_{1}=p^{n-1}-p^{t-1}-\sum_{j=2}^{n-1} p^{j-1} r_{j} .
$$

Equations (6) and (9) imply that

$$
\begin{aligned}
\operatorname{rk}(\mathcal{C}) & =p^{t}+p^{n-1}-p^{t-1}-\sum_{j=2}^{n-1} p^{j-1} r_{j}+\sum_{j=2}^{n-1} r_{j} \\
& =p^{t}+p^{n-1}-p^{t-1}-\sum_{j=2}^{n-1}\left(p^{j-1}-1\right) r_{j}
\end{aligned}
$$


Thus $\operatorname{rk}(\mathcal{C})$ is maximum if $\sum_{j=2}^{n-1}\left(p^{j-1}-1\right) r_{j}=0$ and so $r_{j}=0$ for $j, 2 \leqslant j \leqslant n-1$. Therefore

$$
\operatorname{rk}(\mathcal{C}) \leqslant p^{n-1}+p^{t-1}(p-1) .
$$

On the other hand, the rank of the $p$-scheme constructed in Example 6 is equal to $p^{n-1}+$ $p^{t-1}(p-1)$. Thus

$$
\mathbf{u}(t, p, n)=p^{n-1}+p^{t-1}(p-1) .
$$

This completes the proof of the theorem.

\subsection{Wreath power}

Let $T_{p}=(V, \mathcal{R})$. Since $|\mathcal{R}|=p$, let $\mathcal{R}=\left\{R_{0}, R_{1}, \ldots, R_{p-1}\right\}$. By induction we construct the scheme $\left(T_{p}\right)^{2 n}$, and its valency pattern is as the following form

$$
\mathrm{VP}\left(\left(T_{p}\right)^{2 n}\right)=\left(\begin{array}{ccccc}
1 & p & p^{2} & \ldots & p^{n-1} \\
p & p-1 & p-1 & \ldots & p-1
\end{array}\right)
$$

By Lemma 5, we conclude that $\left\{E_{i}\right\}_{i=0}^{n} \in \operatorname{PE}\left(\left(T_{p}\right)^{2 n}\right)$ is unique. Also for each $X \in V / E_{n-1}$ we have $\left(T_{p}\right)_{X}^{2 n}$ is isomorphic to $\left(T_{p}\right)^{2 n-1}$. For the scheme $\left(T_{p}\right)^{2 n}$ by reordering if necessary, we conclude that the adjacency matrices of each basis relation with valency $p^{n-1}$ is equal to $R_{i} \otimes J_{p^{n-1}}$, for some $0<i \leqslant p-1$; also the adjacency matrices of each basis relation with valency less than $p^{n-1}$ is equal to $I_{p} \otimes S$, where $S$ is one of the basis relations of $\left(T_{p}\right)^{2(n-1)}$.

Theorem 7. Let $n>1$ be an integer and let $p$ be a prime number. Fix $t$ such that $1 \leqslant t<n$. A p-scheme on $p^{n}$ points has the minimum rank $\mathbf{l}(t, p, n)$ if and only if it is isomorphic to $\mathcal{C}^{\prime}\left(T_{p}\right)^{2(n-t)}$, where $\mathcal{C}^{\prime} \in \mathcal{T}_{p^{t}}$. In particular such a p-scheme is schurian.

Proof. Let $\mathcal{C}^{\prime} \in \mathcal{T}_{p^{t}}$, it is clear that the rank of the $p$-scheme $\mathcal{C}^{\prime} \zeta\left(T_{p}\right)^{2(n-t)}$ is equal to $\mathbf{l}(t, p, n)$ which is the minimum rank among all $p$-schemes in $\mathfrak{C}_{p^{n}}$ with thin radical of order $p^{t}$.

Conversely, suppose $\mathcal{C} \in \mathfrak{C}_{p^{n}}$ with the set of basis relations $\mathcal{R}$ such that $m(\mathcal{C})=t$ and $\operatorname{rk}(\mathcal{C})=p^{t}+(n-t)(p-1)$. If $n=2$ then $t=1$ and the result is true. So we can proceed by induction on $n$. Assume $n \geqslant 3$. From (5), (7) and (8) in the proof of Theorem 1, we conclude that for each $j, 1 \leqslant j<t, r_{j}^{\prime}=0$ and for each $j, t \leqslant j \leqslant n-2, r_{j}^{\prime}=1$. Therefore,

$$
r_{j}= \begin{cases}0, & 1 \leqslant j \leqslant t-1 \\ p-1, & t \leqslant j \leqslant n-1\end{cases}
$$

and so the valency pattern of $\mathcal{C}$ is

$$
\operatorname{VP}(\mathcal{C})=\left(\begin{array}{ccccc}
1 & p^{t} & p^{t+1} & \ldots & p^{n-1} \\
p^{t} & p-1 & p-1 & \ldots & p-1
\end{array}\right)
$$


Let $\left\{E_{i}\right\}_{i=0}^{n} \in \operatorname{PE}(\mathcal{C})$ and $X \in V / E_{n-1}$. Then $\mathcal{C}_{X}$ is a $p$-scheme on $p^{n-1}$ points and from Lemma 5 we have

$$
E_{n-1}=\bigcup_{d(R) \leqslant p^{n-2}} R
$$

Hence the valency pattern of $\mathcal{C}_{X}$ is as follows

$$
\operatorname{VP}\left(\mathcal{C}_{X}\right)=\left(\begin{array}{ccccc}
1 & p^{t} & p^{t+1} & \ldots & p^{n-2} \\
p^{t} & p-1 & p-1 & \ldots & p-1
\end{array}\right)
$$

and so $\operatorname{rk}\left(\mathcal{C}_{X}\right)=p^{t}+(n-1-t)(p-1)$. So induction shows that there is $\mathcal{C}^{\prime} \in \mathcal{T}_{p^{t}}$ such that

$$
\mathcal{C}_{X} \simeq \mathcal{C}^{\prime} \chi\left(T_{p}\right)^{2(n-1-t)}
$$

Now, let $S \in \mathcal{R}$. If $d(S) \leqslant p^{n-2}$, then from (10) it follows that $S \subseteq E_{n-1}$ and so

$$
S=I_{p} \otimes S_{X}
$$

If $d(S)=p^{n-1}$, then $S \nsubseteq E_{n-1}$ and so $S_{X}=\emptyset$. Since $\mathcal{C}_{V / E_{n-1}}$ is a thin scheme on $p$ points, there exists exactly one $Y \in V / E_{n-1}, Y \neq X$ such that $S_{X, Y} \neq \emptyset$. Therefore, from $|X|=|Y|=p^{n-1}$ and $d(S)=p^{n-1}$ we conclude that $X \times Y \subseteq S$. This shows that

$$
S=S_{V / E_{n-1}} \otimes J_{p^{n-1}}
$$

From (12) and (13) it follows that $\mathcal{C}$ is isomorphic to the wreath product of $\mathcal{C}_{X}$ and $\mathcal{C}_{V / E_{n-1}}$. Since $\mathcal{C}_{V / E_{n-1}}$ is a thin scheme on $p$ points so it is isomorphic to $T_{p}$. The latter along with (11) implies that $\mathcal{C} \simeq \mathcal{C}^{\prime} \zeta\left(T_{p}\right)^{2(n-t)}$.

For the last statement, the schemes $\mathcal{C}^{\prime}$ and $T_{p}$ are schurian and so is $\mathcal{C}^{\prime} \chi\left(T_{p}\right)^{2(n-t)}$. This completes the proof of the theorem.

\section{3}

Let $\mathcal{C}=(V, \mathcal{R})$ be an association scheme, and $E \in \mathcal{E}(\mathcal{C})$. According to [6, Definition 2.2] we may order the elements of $V$ as follows: if the elements of $V$ are ordered in such a way that for any $u, v \in V$ such that $u, v \in X$ for some $X \in V / E, u$ and $v$ are ordered exactly in the same way as in $X$, then we say that the elements of $V$ are ordered according to the equivalence $E$.

\section{Proof of Theorem 3.}

We prove the theorem by using induction on $n$. For $n=1$, the result is clear. Suppose that the statement holds for any $p$-scheme of degree $p^{n-1}$. Let $\mathcal{C}=(V, \mathcal{R})$ be a $p$-scheme of degree $p^{n}$ and let $\left\{E_{i}\right\}_{i=0}^{n} \in \operatorname{PE}(\mathcal{C})$.

The scheme $\mathcal{C}_{V / E_{n-1}}$ is a thin scheme on $p$ points, let $V / E_{n-1}=\left\{X_{0}, X_{1}, \ldots, X_{p-1}\right\}$. By reordering of elements of $V$ if necessary, we can consider that the elements of $V$ are 
ordered according to the equivalence $E_{n-1}$. But $E_{n-2} \subseteq E_{n-1}$, so each $X_{i}, 0 \leqslant i \leqslant p-1$, is equal to the union of $p$ blocks of $V / E_{n-2}$. Thus by reordering of elements of $X_{i}$ if necessary, we can consider that the elements of $X_{i}$ are ordered according to the equivalence $E_{n-2}$. In this case the elements of $V$ are ordered according to the equivalences $E_{n-2}$ and $E_{n-1}$, simultaneously. In this way by using $\left\{E_{i}\right\}_{i=0}^{n}$ we can consider that the elements of $V$ are ordered according to the all of equivalences $E_{i}, 1 \leqslant i \leqslant n-1$. But $d\left(E_{1}\right)=p$ and $\mathcal{C}_{Y / E_{0}}=\mathcal{C}_{Y}$ is a thin scheme on $p$ points for each $Y \in V / E_{1}$.

Therefore we can see that by the above reordering on $V$, without loss of generality, $V$ is equal to cartesian product of $p$ copies of a set with $p$ elements. Thus we consider $\left(T_{p}\right)^{2 n}$ on the set $V$ with the above ordering.

Suppose $\left(T_{p}\right)^{2 n}=(V, \mathcal{P})$. It is sufficient to show that each element of $\mathcal{P}$ is equal to a union of some elements of $\mathcal{R}$. Let $\left\{F_{i}\right\}_{i=0}^{n}$ be the unique element of $\operatorname{PE}\left(\left(T_{p}\right)^{2 n}\right)$. It is easy to see that for all $i, 0 \leqslant i \leqslant n$ we have $V / F_{i}=V / E_{i}$, and also from Lemma 4 we have $d\left(F_{i}\right)=d\left(E_{i}\right)=p^{i}$. Since $\left(T_{p}\right)_{V / F_{n-1}}^{2 n}$ and $\mathcal{C}_{V / E_{n-1}}$ are $p$-schemes on $p$ points, without loss of generality we may assume that

$$
\left(T_{p}\right)_{V / F_{n-1}}^{2 n}=\mathcal{C}_{V / E_{n-1}}
$$

On the other hand for each $Y \in V / F_{n-1}$, we have

$$
\left(\left(T_{p}\right)^{2 n}\right)_{Y} \simeq\left(T_{p}\right)^{2(n-1)} .
$$

Now let $X \in V / E_{n-1}$, then $\mathcal{C}_{X}$ is a $p$-scheme on $p^{n-1}$ points. From the hypothesis of the induction, it follows that

$$
\left(T_{p}\right)^{2(n-1)} \leqslant \mathcal{C}_{X} .
$$

By (15) and (16), for each $P \in \mathcal{P}$ with $d(P) \leqslant p^{n-2}$, there exists a subset $\mathcal{D}_{P} \subseteq \mathcal{R}$ such that

$$
P=\bigcup_{R \in \mathcal{D}_{P}} R
$$

Now we want to show that each relation of $\mathcal{P}$ with degree $p^{n-1}$ is also a union of some elements of $\mathcal{R}$. Let $V / E_{n-1}=\left\{X_{1}, X_{2}, \ldots, X_{p}\right\}$ be the set of points of the $p$-scheme $\mathcal{C}_{V / E_{n-1}}$. Any $\hat{R}:=R_{V / E_{n-1}} \in \mathcal{R}_{V / E_{n-1}}$ is corresponding to a permutation $g_{\hat{R}}$ on the set $V / E_{n-1}$ as follows:

$$
X_{i}^{g_{\hat{R}}}=X_{j} \Longleftrightarrow R_{X_{i}, X_{j}} \neq \emptyset \text {. }
$$

Define a binary relation $\sim$ on $\mathcal{R}$ as follows:

$$
R, S \in \mathcal{R}, \quad R \sim S \Longleftrightarrow \hat{R}=\hat{S} .
$$

Clearly, $\sim$ is an equivalence relation on $\mathcal{R}$. Let $[R]$ be the congruent class containing $R$. It is clear that $T \in[R]$ if and only if $g_{\hat{T}}=g_{\hat{R}}$. Also define $\mathcal{U}:=\left\{\left[R_{k}\right], 0 \leqslant k \leqslant p-1\right\}$ the set of all distinct congruent class of relation $\sim$ on $\mathcal{R}$ such that $\left[R_{0}\right]=\left\{R \in \mathcal{R}: R_{X_{i}, X_{i}} \neq\right.$ $\emptyset, 1 \leqslant i \leqslant p\}$.

Let $\left[R_{k}\right] \in \mathcal{U}, 0 \leqslant k \leqslant p-1$, and $X_{i}, X_{j} \in V / E_{n-1}$ such that $R_{k} \cap X_{i} \times X_{j} \neq \emptyset$. For each $(x, y) \in X_{i} \times X_{j}$, there exists a basis relation $T \in \mathcal{R}$ such that $(x, y) \in T$ and so 
$X_{i}^{g_{\hat{T}}}=X_{j}$. Therefore, $T \in\left[R_{k}\right]$. This shows that $X_{i} \times X_{j} \subseteq \bigcup_{T \in\left[R_{k}\right]} T$. Then it follows that

$$
\bigcup_{T \in\left[R_{k}\right]} T=\bigcup_{X_{i}^{g} \hat{R}=X_{j}} X_{i} \times X_{j}
$$

and so

$$
\bigcup_{T \in\left[R_{k}\right]} T=P_{g_{R_{k}}} \otimes J_{p^{n-1}}
$$

where $P_{g_{\hat{R}_{k}}}$ is a permutation matrix correspond to $g_{\hat{R}_{k}}$.

Let $P \in \mathcal{P}$ with $d(P)=p^{n-1}$, then $P$ is equal to tensor product of an adjacency matrix of a non diagonal basis relation of $T_{p}$ with $J_{p^{n-1}}$. From equations (14) and (18) we conclude that for each $P \in \mathcal{P}$ with $d(P)=p^{n-1}$ there exists a class $\left[R_{k}\right] \in \mathcal{U}, 0<k \leqslant p-1$ such that

$$
P=\bigcup_{T \in\left[R_{k}\right]} T .
$$

By equations (17) and (19), the proof is complete.

\section{Acknowledgements}

The authors would like to thank Professor Ilia Ponomarenko for his valuable arguments in Example 6.

\section{References}

[1] K. Kim. Characterization of $p$-schemes of prime cube order. J. Algebra, 331:1-10, 2011.

[2] M. Muzychuk. A wedge product of association schemes. European J. Combin., 30:705-715, 2009.

[3] I. N. Ponomarenko. Cellular algebras and graph isomorphism problem. Research report No. 8592-CS, university of Bonn, 1993.

[4] I. N. Ponomarenko and A. Rahnamai Barghi. On the structure of $p$-schemes. $J$. Math. Sci., 147(6):7227-7233, 2007.

[5] A. Rahnamai Barghi and I. N. Ponomarenko. The basic digraphs of $p$-schemes. Graphs Combin., 25:265-271, 2009.

[6] B. Xu. Characterizations of wreath products of one-class association schemes. $J$. Combinatorial Theory Ser. A, 118:1907-1914, 2011.

[7] P.-H. Zieschang. Theory of association schemes. Springer, Berlin, Heidelberg, 2005. 